\title{
Development and characterization of a $z$-pinch driven hohlraum high-yield inertial confinement fusion target concept
}

\author{
Michael E. Cuneo, Roger A. Vesey, John L. Porter, Jr., Gordon A. Chandler, \\ David L. Fehl, Terrance L. Gilliland, David L. Hanson, John S. McGurn, \\ Paul G. Reynolds, Lawrence E. Ruggles, Hans Seamen, Rick B. Spielman, \\ Ken W. Struve, William A. Stygar, Walter W. Simpson, Jose A. Torres, \\ David F. Wenger \\ Sandia National Laboratories, Albuquerque, New Mexico 87185-1193 \\ James H. Hammer, Peter W. Rambo \\ Lawrence Livermore National Laboratories, Livermore, California 94551 \\ Darrell L. Peterson, George C. Idzorek \\ Los Alamos National Laboratories, Los Alamos, New Mexico 87545
}

\begin{abstract}
We describe the initial experiments to study the Z-pinch-driven hohlraum high-yield inertial confinement fusion (ICF) concept of Hammer and Porter [J. H. Hammer et al., Phys. Plasmas, 6, 2129(1999)]. We show that the relationship between measured pinch power, hohlraum temperature, and secondary hohlraum coupling ("hohlraum energetics") is well understood from 0-D semi-analytic, 2-D viewfactor, and 2-D radiation magneto-hydrodynamics models. These experiments have shown the highest $\mathrm{x}$-ray powers coupled to any $\mathrm{Z}$-pinch driven secondary (26 $\pm 5 \mathrm{TW}$ ), indicating the concept could scale to fusion yields of $400 \mathrm{MJ}$. We have also developed a novel, single-sided power feed, double-pinch driven secondary that meets the pinch simultaneity requirements for polar radiation symmetry. This source will permit investigation of the pinch power balance and hohlraum geometry requirements for ICF relevant secondary radiation symmetry, leading to a capsule implosion capability on the $\mathrm{Z}$ accelerator $[R$. B. Spielman, et al., Phys. Plasmas. 5, 2105 (1998)].
\end{abstract}




\section{Introduction}

Wire array $\mathrm{Z}$-pinches ${ }^{1-3}$ on the $\mathrm{Z}$ accelerator ${ }^{3}$ are the most intense laboratory source of soft $\mathrm{x}$-rays in the world. This source provides an extended, nearly-Planckian radiation source with: high $x$-ray production efficiency (10-15\% wall plug), large $x$-ray powers and energies $(\geq 100$ $\mathrm{TW},>0.7 \mathrm{MJ}$ in $7 \mathrm{~ns}$ ), large characteristic hohlraum volumes (up to $10 \mathrm{~cm}^{3}$ ), long pulse-lengths ( 5 to $15 \mathrm{~ns}$ ), $\mathrm{x}$-ray energy scaling with $\mathrm{I}^{2}$ through $20 \mathrm{MA}$, good reproducibility (better than $\pm 10 \%$ demonstrated) and low cost ( $25 \$ /$ radiated Joule). This unique combination of characteristics may make Z-pinch $\mathrm{x}$-ray sources a good match to the requirements for indirect drive inertial confinement fusion (ICF) of high-yield scale ( $>200$ MJ fusion yield) capsules if adequate radiation temperature, symmetry, pulseshape and capsule preheat conditions can be attained ${ }^{+}$. We describe initial experiments to characterize the pinch power, hohlraum radiation temperatures, and secondary radiation coupling efficiency for the Z-pinch driven hohlraum (ZPDH) approach of Hammer and Porters. We have also developed a single-sided power feed, double-pinch driven secondary capability that will permit polar radiation symmetry and capsule implosion experiments on the $\mathrm{Z}$ accelerator. We note that other indirect-drive ICF schemes have also been studied using a $\mathrm{z}$-pinch $\mathrm{x}$-ray source ${ }^{6.7}$.

This concept is depicted in Fig. 1. In this concept, two Z-pinch-driven primary hohlraums are located at either end of a coaxial secondary hohlraum containing a fusion capsule ${ }^{5}$. This topology of coupling two hot $\mathrm{x}$-ray source regions to a cooler capsule secondary is similar to some laser-driven hohlraum configurations ${ }^{4.3}$. Each primary hohlraum is driven by the implosion of a wire array consisting of hundreds of uniformly-spaced fine $\mathrm{W}$ wires. Electromagnetic power enters each primary through a small ( $2 \mathrm{~mm}$ wide) annular anode-cathode (AK) gap at the base of the wire array. The AK gaps are coupled to a single pulsed-power driver through their own set of 


\section{DISCLAIMER}

This report was prepared as an account of work sponsored by an agency of the United States Government. Neither the United States Government nor any agency thereof, nor any of their employees, make any warranty, express or implied, or assumes any legal liability or responsibility for the accuracy, completeness, or usefulness of any information, apparatus, product, or process disclosed, or represents that its use would not infringe privately owned rights. Reference herein to any specific commercial product, process, or service by trade name, trademark, manufacturer, or otherwise does not necessarily constitute or imply its endorsement, recommendation, or favoring by the United States Government or any agency thereof. The views and opinions of authors expressed herein do not necessarily state or reflect those of the United States Government or any agency thereof. 


\section{DISCLAIMER}

Portions of this document may be illegible in electronic image products. Images are produced from the best available original document. 
low-inductance magnetically-insulated-transmission-lines ${ }^{3}$ which deliver large currents to the wire arrays. The wall of the primary hohlraum is part of the current feed path (current return electrode), but does not move significantly because of the large mass difference between it and the pinch. Z-pinch driven primaries of the same scale as that in the proposed system (see Fig. 2B) have reached radiation temperatures of $140 \pm 7 \mathrm{eV}$ when driven by $20 \mathrm{MA} Z$-pinches producing $131 \pm 33 \mathrm{TW}^{9,10}$. The pinch is imploded onto foam shells, foam cylinders or internal, nested wire arrays to provide pulse shaping ${ }^{5,7}$. Radiation is transported into the secondary hohlraum through a transparent Beryllium radial-spoke-electrode and shine shield that isolates the capsule from the pinch plasma, magnetic field, and direct $\mathrm{x}$-ray shine. These spokes carry the pinch current during the implosion process, and confine pinch plasma to the primary hohlraum region, but allow transport of $\mathrm{x}$-rays into the secondary in between the spokes. The coupled hohlraums provide two levels of radiation smoothing over the mm-scale-length non-uniformities typical of Z-pinches. Viewfactor calculations ${ }^{5,11}$ show that the majority of the secondary drive comes from primary wall re-radiation ( $>70 \%$ of the flux entering the secondary). Less than $30 \%$ of the secondary wall drive comes from direct-pinch-illumination. The capsule does not directly view the pinch. The control of symmetry is accomplished globally or geometrically in the ZPDH by overlapping radiation sources from the primary and secondary hohlraum walls on the capsule to create a uniform drive ${ }^{5.11}$. The possibility of global or geometric symmetry control is permitted because the pinch is an extended radiator. Viewfactor and 2-D radiation magneto-hydrodynamic (RMHD) simulations show that radiation symmetry adequate for high-convergence capsule implosions can be obtained via geometric control. The ZPDH approach places minimal emphasis on Z-pinch uniformity and stability, and therefore has minimal reliance on control of the highenergy-density plasma physics of the Z-pinch. De-coupling the Z-pinch source region from the

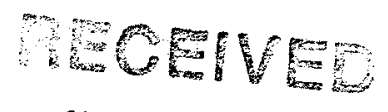

NOY 152000

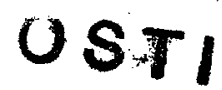


capsule implosion promises an approach that is more readily modeled with available 2-D codes, and therefore has improved predictability to scale the system.

The reader is referred to the previously published design study for details of the high-yield capsule design and system requirements ${ }^{5}$. The experiments described in this paper characterize the hohlraum energetics, and secondary coupling at the $20 \mathrm{MA}$ drive current level. Other issues which must be studied in the future are the scaling of pinch power and power-flow coupling with drive current and hohlraum temperature, polar and azimuthal radiation symmetry, pulseshaping, and capsule preheat. The diagnostics and experimental layout are described in section II. Characterization of the pinch $\mathrm{x}$-ray power, energy and spectrum including the effects of energy loss at the AK gap are described in section III. Assessment of the hohlraum radiation temperature and wall re-emission spectrum, the hohlraum energetics balance and measurement and modeling of the radiation coupling efficiency (spoke transparency) are discussed in section IV. Selected results from a single-sided power feed, two-sided or double-pinch driven secondary are discussed in section V. We summarize and provide a future outlook in section VI.

\section{Diagnostic description and experimental configurations}

Spatially-integrating $\mathrm{x}$-ray detectors of various types view the $\mathrm{x}$-ray flux emitted from the Au hohlraum walls of the primary and secondary through a 3 or $4 \mathrm{~mm}$ diameter aperture, or from a fraction (40-50\%) of the pinch height. Time and spatially-resolved, filtered $\mathrm{x}$-ray framing pinhole cameras (FC) measure the size of the hohlraum wall apertures with 4 filter levels between 250 and $850 \mathrm{eV}^{12}$ to correct for aperture closure. The $\mathrm{Au}$ apertures are tamped by a 20 $\mu \mathrm{m}$ coating of Parylene-N. The measured radial closure in the primary/secondary configuration at peak radiation temperature is $0.52 \pm 0.04 \mathrm{~mm}(54 \pm 3 \%$ open for $4 \mathrm{~mm}$ apertures) for $127 \pm 8 \mathrm{eV}$ 
in the primary, and $0.30 \pm 0.05 \mathrm{~mm}(72 \pm 6 \%$ open for $4 \mathrm{~mm}$ apertures) for $89 \pm 5 \mathrm{eV}$ in the secondary. All wall temperatures shown in this paper have been closure corrected for the measured aperture size at the time of peak temperature.

Our principal hohlraum wall temperature diagnostic for both the primary and secondary are $\mathrm{x}$-ray transmission grating spectrometers $(\mathrm{TGS})^{13}$, which provide a time-resolved measurement of the wall re-emission spectra with Si PIN diode detectors over 10 to 16 different energy bins between 125 and $900 \mathrm{eV}$. Wall temperature histories both from the primary and secondary are extracted by Planckian curve fits to the unfolded spectra, which show highly-thermal distributions. A five channel, filtered carbon cathode $\mathrm{x}$-ray diode $(\mathrm{XRD})^{14}$ array and a seven channel filtered Si PIN detector array $\left(\right.$ FSDA) ${ }^{15}$ also views the secondary hohlraum wall through the same aperture as the TGS and FC (see Fig. 2A). The total x-ray flux from the hohlraum wall was also measured through this aperture with a $1 \mu \mathrm{m}$ thick nickel bolometer (BOL) with nearly intrinsic resistivity values ${ }^{16}$. A six channel filtered imaging Si PIN detector array (ISDA) was also used. This diagnostic collects from a $200 \times 200 \mu \mathrm{m}^{2}$ spatial element near the center of the aperture to eliminate the necessity of aperture closure corrections.

Hohlraum wall brightness temperatures are also obtained from the measured bolometer energy by using various $\mathrm{x}$-ray detector signals as flux shape factors. Using a Si PIN detector from the TGS Si PIN array chosen at the peak energy of the particular Planckian spectra shows agreement with the peak unfolded TGS temperatures within $+1.5 \pm 2.6 \%$ ( 7 values). The peak brightness temperatures of the FSDA (assuming a Planckian) agree with the TGS values within $-2.3 \pm 4 \%$ ( 4 values). Using the $4 \mu \mathrm{m}$ Kimfoil filtered XRD as a bolometer shape factor (140-280 $\mathrm{eV}$ ) overestimates the temperature compared to the TGS unfold by $+5.2 \pm 3.6 \%$ ( 7 values). Uncertainty of the temperature measurements is therefore no larger than $\pm 5 \%( \pm 20 \%$ in flux $)$ 
based on combined unfold, instrumental and aperture closure uncertainty, confirmed by three independent diagnostics. This is comparable to those for laser hohlraums ${ }^{4}$.

The Z-pinch $\mathrm{x}$-ray spectrum, power, and energy are also measured by an independent XRD array, bolometers, and a TGS looking through $6 \mathrm{~mm}$ wide slots in the current return can. This configuration requires no aperture closure correction. The pinch is also sometimes viewed through a $4 \mathrm{~mm} \times 4 \mathrm{~mm}$ tamped aperture on the side of a hohlraum can. The signal is corrected at peak by the measured aperture closure (lengthwise), which gives an upper bound on pinch power. The closure may be overestimated because the measured aperture edge motion is backlit by the $120 \mathrm{eV}$ wall re-emission rather than the hotter $165 \mathrm{eV}$ pinch. Pinch powers are obtained from absolute unfolds of the 5-channel XRD array ${ }^{14}$, and unfolds of TGS, and can also be estimated by using the two lowest energy filtered (kimfoil and vanadium) XRD channels as shape factors for the bolometer energy and then averaging. Uncertanties in pinch power measurements are discussed in section IV. Power flow in the transmission line feed is characterized with B-dot current monitors ${ }^{17}$.

Fig. 2 shows the configuration and diagnostic layout for the experiments described in this paper. Experiments characterizing the pinch output and showing sensitivity of radiation pulseshape to current return electrode geometry (Fig. 2A, 2B) are described in section III. Primary to secondary hohlraum coupling experiments were performed in the single-sided system (Fig. 2A). These are described in section IV. Double pinch experiments with a single-current feed (Fig. 2C) are described in section V. In this geometry the transmission line power feed is extended along the outside of the secondary to provide current to an upper wire array. The proposed high yield geometry discussed in Fig. 1 (dotted lines in Fig. 2A) requires a machine architecture permitting a two-sided power feed. 


\section{Pinch energetics and power flow}

The pinch $x$-ray pulseshape (risetime and FWHM) shows sensitivity at the $15-25 \%$ level (12 ns) to the location and size of diagnostic apertures in the return current electrode (the primary hohlraum wall), and to the method of positioning and tensioning the wires. Apertures in the side of the hohlraum wall of order 3 to $4 \mathrm{~mm}$ in size are located within $2 \mathrm{~mm}$ of the initial wire array position to minimize hohlraum wall area and radiation leakage out the AK gap. They thus generate a significant radial magnetic field perturbation near the wires which may cause local $\mathrm{J}_{z} \times \mathrm{B}_{r}=\mathrm{V}_{\theta}$ azimuthal acceleration of the wires during the first half of the implosion. Wires from the array pass around the secondary (e.g. out the top of the primary, see Fig. 2A), and thus must be moved out of the way of diagnostic apertures. This is performed with a device called a wirespreader which moves the wires azimuthally. Wires are displaced at the top of the wire array by up to $35 \mu \mathrm{m}$ azimtuhally from an ideal centered position by a wire-spreader. Non-uniform azimuthal spacing of wires may act as a seed for wire-wire filamentation instability ${ }^{18}$. Both of these hypotheses are under investigation. Normalized pulseshapes showing examples of the effects of aperture size and placement and azimuthal wire position are plotted in Fig. 3, where the $50 \%$ point has been shifted to overlay at $100 \mathrm{~ns}$. Consider the pulseshape from an average of 5 shots with a solid primary (Fig. 2B) with minimal perturbation by apertures (setup A), wire placement, and no Be spokes as nominal in Fig. 3. The pulseshape from shots with a symmetrically slotted return-current can with and without Be spokes (not shown in Fig. 3, but see Table I below) closely overlay this shape and FWHM within the typical variation shown by

the error bars. This indicates that the Be spokes do not have a mahor effect on pulseshape. The pulseshape from shot 643 with a primary/secondary configuration (see Fig. 2A) with spokes and a somewhat different primary aperture setup (setup B) is somewhat narrower and has a faster 
risetime by comparison, when wire spreader hardware is not used. This comparison also indicates that the Be spokes do not have a major effect on pulseshape. Compare now shot 644 , which is the same as 643 except for the use of wire spreader hardware. The FWHM increases from 5.9 to $7.3 \mathrm{~ns}$, and the risetime increases from 4.2 to $5.3 \mathrm{~ns}$. The average of 3 primary/secondary shots with spreader hardware and a different aperture setup (setup $\mathrm{C} 1$ ) is very similar to this case. This is the pinch power pulseshape driving hohlraum energetics experiments in section IV. Aperture setup C2 increased the azimuthal extent of an aperture from $4 \mathrm{~mm}$ to 6 $\mathrm{mm}$ (shot 499 , with spreader). Although the shape of the pulse risetime is the same, the FWHM increased from 7.7 ns to $9.5 \mathrm{~ns}$. In this case, both wire-spreader and aperture effects broaden the pulse. These effects are clearly second-order 3-D effects ( $r-\theta$ plane physics) on pinch performance. Experiments in other geometries (see section V) confirm the broadening effects of large, asymmetric apertures on FWHM and the effect of differences in wire positioning and tensioning. Table I summarizes results of pinch characterization from several different diagnostics (BOL, XRD shape factor + BOL, XRD unfold, and TGS) for 4 different geometries. A symmetric current return electrode (slots or symmetric aperture setups A and B) with no spreader, provided a $129( \pm 7$ relative $)( \pm 32$ absolute $) \mathrm{TW}, 1160( \pm 25)( \pm 230) \mathrm{kJ}, 6.4 \pm 0.4 \mathrm{~ns}$ radiation pulse. A hohlraum with asymmetric diagnostic apertures (e.g. setup $\mathrm{Cl}$ from primary in Fig. 2A) and/or wire spreader hardware, provided $100( \pm 6)( \pm 25) \mathrm{TW}, 1042( \pm 15)( \pm 208) \mathrm{kJ}$, and $7.5 \pm 0.6 \mathrm{~ns}$. These pulses represent a $10 \pm 2 \%$ conversion efficiency of total stored energy to radiation. We note that the pinch implosion time is identical with and without Be spokes to within $\pm 2 \%$.

Figure 4 compares the measured currents (near peak) and pinch powers from shot 566 with results from a 0-D circuit model and a 2-D $(r-z)$ Eulerian radiation magneto-hydrodynamics 
simulation (E-RMHD) with random seeds to initiate the pinch magneto-Rayleigh-Taylor (RT) instability growth ${ }^{19.20}$. Drive currents are measured upstream $\left(\mathrm{I}_{\text {total }}\right)$ and downstream $\left(\mathrm{I}_{\text {conv }}\right)$ of the transmission line series current adder (a "current convolute") ${ }^{3.17}$. The drive currents rise linearly over 100 ns to peak amplitude. The last $35 \mathrm{~ns}$ is shown in Fig. 4. Although the post-convolute current monitor is $5 \mathrm{~cm}$ from the load, we do not observe the strong reduction in load current accompanying the inductance change of the pinch near stagnation (compare $\mathrm{I}_{\text {load } 0-\mathrm{D}}$ or $\mathrm{I}_{\text {load2-D }}$ with $\left.I_{\text {conv }}\right)$. There are several possible explanations. The B-dot measurements are believed to be accurate to within $\pm 5 \%$ at peak. It is possible that the B-dots are less reliable after peak as the electron dose increases. Figure 4 gives a 7 shot average of these currents. The overall shape of the total and convolute currents is similar, suggesting nominal performance. A 3-D implosion (as compared to the 0-D thin shell limit or a 2-D model) would limit the inductance change, and soften the change in slope. Note on Fig. 4 for example, that the slope of $I_{\text {load2-D }}$ is somewhat smaller than for $I_{\text {loado-D }}$. The effect of current return electrode azimuthal symmetry and uniformity of wire spacing on X-ray FWHM discussed above suggests 3-D effects ( $r$ - $\theta$ plane physics) are important. A third possibility proposed in previous work ${ }^{20}$ is that current loss at the AK gap in parallel with the load could increase the convolute current by lowering the downstream impedance, preventing observation of the inductive spike. We indirectly infer the presence of current loss at this AK gap by comparing the convolute current on shots with different AK gaps. Shots with larger AK gaps show lower convolute currents implying larger downstream impedance and therefore less parallel current loss. A time-dependent impedance representing electron loss at the base of the pinch was obtained from 0-D circuit simulations which reproduce the measured currents through 225 ns. This impedance was included in parallel with the load in the 2-D E-RMHD simulation to represent AK gap current loss. This impedance does increase 
$I_{\text {conv2-D }}$ towards the experimental value in Fig. 4, but is apparently too high after about $225 \mathrm{~ns}$. Further iterations could be done on the parallel impedance to reproduce the convolute current. See reference 20 for an example of this optimization

The 2-D pinch power is scaled by the ratio $\left(\mathrm{I}_{\text {conv }} / \mathrm{I}_{\text {conv2-D }}\right)^{2} \approx 0.95$ to account for the current difference between simulation and experiment. We find reasonable agreement between the scaled 2-D simulation $\left(P_{2 D}=131 \mathrm{TW}\right)$ with the measured power $\left(\mathrm{P}_{\mathrm{XRD} 566}=127 \mathrm{TW}\right)$ for shot 566 (symmetric slotted can, no spokes), for a $3 \%$ random seed. A 2-D x-ray energy of $850 \mathrm{~kJ}$ is predicted prior to the time when the code energy conservation becomes marginal, which agrees with the energy from shot 566 to the same point in the power pulse. This is also the same period where the pulse is useful for driving ICF applications, i.e. during the main pulse, not including the tail. The $0-\mathrm{d}$ thin shell model gives $650 \mathrm{~kJ}$ of kinetic energy through a $20: 1$ convergence ratio, about $50 \%$ less than the $1000 \mathrm{~kJ}$ total observed. Analysis of the details of the 2-D simulation suggest the additional energy is PdV work by the magnetic field on the plasma ${ }^{19.20}$. Thermalization of the implosion kinetic energy occurs through shock heating, which produced about $625 \mathrm{~kJ}$ of internal energy at the time of $850 \mathrm{~kJ}$ radiated. PdV work by the magnetic field on the pinch plasma produces an additional $300 \mathrm{~kJ}$ of internal energy through this time. Ohmic heating contributes $50 \mathrm{~kJ}$ of internal energy for a total of $975 \mathrm{~kJ}$. About $125 \mathrm{~kJ}$ remains trapped in specific heat and is not radiated. Energy output of the 2-D simulations is insensitive to the value of the random seed ${ }^{19.20}$. In this simulation, current loss decreased load current by about 1-2 MA (compare $I_{\text {conv2-D }}$ and $I_{\text {noloss }}$ ) which produced a $30 \%$ reduction in scaled pinch power and energy. A case without current loss produces about $169 \mathrm{TW}\left(\mathrm{P}_{2 \text { Dnoloss }}\right)$ and $1120 \mathrm{~kJ}$ during the same part of the pulse, for the same random seed. Larger random seeds would be required in this 2-D code to reproduce the larger FWHM and lower power shots from 3-D effects discussed 
above. If this analysis is correct, we expect that larger radiated energies would be possible with larger AK gaps.

Figure 5 shows the pinch energy spectrum at peak power from a TGS unfold for shot 567 , compared with predictions from a 2-D Lasne ${ }^{21}$ E-RMHD pinch simulation. Since the pinch size is unknown, we perform a two-parameter Planckian fit to the measured spectrum, varying effective source area and temperature. The TGS unfold shows a nearly-Planckian spectrum with a temperature of $164 \pm 7 \mathrm{eV}$, and an effective diameter of about $4.8 \pm 0.9 \mathrm{~mm}$. An unfold of the filtered XRD array (not shown) gives a similar Planckian temperature $(171 \pm 26 \mathrm{eV})$ with an effective diameter of about $2.8 \pm 1.5 \mathrm{~mm}$, when the lowest energy channel with the largest unfold uncertainty is neglected. Reasons for this discrepancy are being investigated. Direct measurements of the pinch size with a pinhole camera show a 2-3 mm FWHM at peak power in rough agreement with these observations. The 2-D RMHD simulation results are post-processed to produce a synthetic TGS spectrum which is well represented $\left(9 \% \chi^{2}\right)$ by the sum of two Planckian distributions, each Planckian with its own temperature and area. A single-Planckian fit is a poor representation of the 2-D simulation results $\left(32 \% \chi^{2}\right)$. The hot tail $(301 \mathrm{eV}, 0.00876$ $\mathrm{cm}^{2}$ ) is believed to represent emission from hot spots as RT bubbles reach the axis, while the lower temperature fit to the bulk of the spectrum $\left(136 \mathrm{eV}, 0.356 \mathrm{~cm}^{2}\right)$ is thought to correspond to emission from the RT spikes. More analysis of these results is required. The amount of the spectrum in the hot tail fluctates in time. Fig. 5 shows an intermediate value. The measured spectrum is best represented by a single-Planckian $\left(10 \% \chi^{2}\right)$. Differences may correspond to a more 3-D nature of the pinch implosion compared to the simulations (see discussion in section IV). The Planckian nature of the measured spectrum implies a stagnated plasma with high optical depth. providing an efficient radiator. Using the Roseland mean opacity of $\mathrm{Au}^{+}$to represent that 
of $\mathrm{W}$, we estimate an optical depth of 5-10 for the core of this stagnated pinch ${ }^{10}$. The 2-D simulations presented in Fig. 4 predict a mass averaged pinch diameter of less than $0.5 \mathrm{~mm}$ at peak power, and a length averaged diameter defined by unity opacity of $2.2 \mathrm{~mm}$ at peak power. The experimental observations of $2-5 \mathrm{~mm}$ diameter are consistent with the presence of 3-D effects on pinch implosion.

\section{Hohlraum energetics and secondary radiation coupling}

The hohlraum radiation temperature is determined by a balance of the pinch power with losses to the Au wall ${ }^{4.8}$, out the AK gap or diagnostic apertures, into Be spoke material, into the secondary by radiative coupling between the two hohlraums and by direct shine of pinch radiation on the secondary wall. A single-sided secondary configuration (Fig. 2A) was used to characterize the average Be spoke transparency. We measure the primary and secondary wall reemission temperatures, and the pinch power simultaneously. These data are compared in several hohlraum energetics models for consistency. These treatments are (a: 1) 0-D, 2-region, spatiallyaveraged power-balance model similar to previous analytic treatments ${ }^{4.5 .8}$, but with timedependent wall albedos taken from 1-D RMHD calculations using the measured temperature history $\left.{ }^{10} ; 2\right)$ a 2-D spatially-resolved viewfactor model with local, time-dependent wall albedos pre-calculated as above and parameterized within the code ${ }^{11}$ with a uniform Lambertian static pinch on axis in the primary; and 3) a 2-D RMHD ${ }^{21}$ treatment of the system with a uniform Lambertian collapsing pinch source. Measurement of the primary and secondary temperatures and a pinch power pulse-shape allow each of these models to be solved for peak pinch power and average spoke-region transparency at peak temperature as unknowns. The peak power and 
transparency from these 3 models all agree with each other, and the measured pinch power within the experimental error.

Figure $6 \mathrm{a}$ and $6 \mathrm{~b}$ show a comparison of the primary and secondary temperature measurements with the 2-D viewfactor and two 2-D RMHD models. Very good agreement is obtained between the models and measurements. A peak pinch power of $106+20 /-30 \mathrm{TW}$ was measured (Fig. 6a), after aperture closure correction. The inferred peak pinch power from the 2D viewfactor (VF) simulations was $95 \pm 16 \mathrm{TW}$. The simulation error reflects the $\pm 5 \%$ uncertainty in temperature using $\mathrm{P} \approx \mathrm{T}^{3.6}$ from a $0-\mathrm{D}$ scaling law for the $\mathrm{ZPDH}^{10}$. A transparency of $65 \%$ was obtained for the 2-D VF simulation with the entrance to the secondary fully open. When $\mathrm{Au}$ hohlraum wall motion was approximated by closing the secondary entrance down by $1 \mathrm{~mm}$ on the radial width, a $72 \%$ transparency was required (not shown). We refer to the transparency inferred from the initial secondary entrance area as a total transparency, while that inferred by accounting for entrance closure is a Be transparency. Two 2-D RMHD simulations bound the primary and secondary temperatures: a case with 106 TW (as measured) and 65\% transparency, and a case with $95 \mathrm{TW}$ and $72 \%$ transparency. The best agreement with the primary temperature is for this case. The 2-D RMHD simulations include the radiation driven expansion from the $\mathrm{Au}$ walls, closing the AK gap and secondary entrance. We expected that AK gap closure in the 2-D RMHD case should reduce the power required compared to the 2-D VF case without closure, but did not observe this. Note that to obtain improved agreement with the measured early foot on the radiation drive, a low level power was assumed, rising up to 1 TW over the first 80 ns of the pinch run-in to stagnation. The low pinch power during the 90 ns pinch run-in raises the albedo to of order $0.7-0.8$ prior to the main pulse. This early power cannot be measured since the pinch diameter is larger than the $4 \mathrm{~mm}$ window for most of the run-in. 
Results of the 0-D region-averaged mode $\left.\right|^{5,8,10}$ for power and transparency are similar to the 2-D models. A Lambertian pinch with the AK gap and secondary fully open requires $113 \pm 19$ TW, and $65 \pm 5 \%$ transparency for shot 413 . Larger powers are required as a result of the region averaged nature of the model. The 0-D model is used to study sensitivity of the power and transparency to AK gap closure, closure of the secondary entrance, and to the fraction of pinch power that directly shines into the secondary. The last depends on the pinch angular emission profile. 2-D RMHD simulations including the effects of magnetic-field tamping of plasma expansion show AK gap closure of the Au hohlraum wall by $1.5 \mathrm{~mm}$ out of an initial $2 \mathrm{~mm}$ by peak power. This closure reduces the required power for consistency with the measured temperatures by $10 \%$ and increases the transparency by $3 \%$. Closure of the secondary by of order $0.7 \mathrm{~mm}$ on the radial width of the entrance consistent with 2-D RMHD simulations, reduces required power by $1.4 \%$ and increases transparency by $7 \%$. The more highly non-Lambertian the pinch, the lower the direct shine fraction. Thus more of the pinch power stays in the primary. Assuming a non-Lambertian distribution which is $15 \%$ above a Planckian at 12 degrees, the power is reduced by $5 \%$ and the transparency increased by $11 \%$. The $0-\mathrm{D}$ model also allows us to accurately assess the affect of temperature uncertainty on transparency $( \pm 8 \%)$. An average of 3 shots gives a total transparency of $64 \pm 6 \%$, and a Be transparency of $71 \pm 7 \%$. Total transparencies of more than $60 \%$ are required to scale this approach to high-yield without more than a $20 \%$ driver energy penalty ${ }^{5}$.

We see reasonable agreement between the predicted range of pinch powers $(95 \pm 17$ to $113 \pm 19 \mathrm{TW})$ and the measurement $(106+20 /-30 \mathrm{TW})$. The largest potential uncertainty in assessing energetics balance is the measurement of pinch power. particularly when an aperture closure correction is required as in a hohlraum. The aperture closure correction results in an 
upper bound on pinch power. Hohlraum wall re-emission or reflection of hohlraum radiation field from the pinch can also be measured along the pinch LOS, and may contribute as much as $3-12 \%$ to the signal based on 3-D viewfactor simulations. Pinch diagnostics view the middle 40$50 \%$ of the pinch length at $12^{\circ}$, and assume a spatially uniform, cylindrical pinch that has a uniform angular distribution (Lambertian emitter) to unfold to total power. The angular emission profile from a 2-D RMHD pinch simulation has a brightness $30 \%$ higher than a Lambertian source when measured along an LOS that is angled at $12^{\circ}$ from the pinch midplane. At shallow angles, the $\mathrm{x}$-rays are collected from the hotter RT bubbles stagnating on axis which are not attenuated by the surrounding cooler RT spike structure. This may account for the non-Planckian nature of the spectrum from the simulation presented in Fig. 5. The pinhole photographs and other evidence presented above indicate a clear 3-D structure, which would reduce the nonLambertian, and non-uniform nature of the angular emission predicted in the 2-D simulations. Xray pinhole framing camera pictures of the pinch self-emission (see Fig. 2) however, also show a spatially non-uniform pinch, and we cannot rule out cooler emission from the ends of the pinch. The net effect of non-uniform and non-Lambertian emission would be to decrease the total energy and power, perhaps by as much as $15 \%$. Finally, there is the unfold and calibration uncertainty. We assume a net uncertainty on directly measured pinch power of $+20 \% /-30 \%$ from all these effects. The assumption of a uniform Lambertian pinch has been satisfactory for near term pinch physics experiments because the absolute pinch powers were not as important as relative performance. We must address the possible systematic errors because we desire an absolute hohlraum power balance. The hohlraum acts as an integrating sphere, smoothing and integrating over the pinch spatial nonuniformities and $\mathrm{x}$-ray angular distribution. As noted above, $\mathrm{a} \pm 5 \%$ absolute temperature uncertainty corresponds to $\mathrm{a} \pm 17 \%$ absolute power uncertainty. The 
measurement of the wall temperature may be the best measurement of the effective pinch power, within the uncertainties of wall albedo and the range of uncertainty given by the unknown amount of AK gap closure (10-15\% in flux).

We observe radiation spectral density from both primary and secondary hohlraum walls that are nearly-Planckian from $5-10 \mathrm{~ns}$ prior to peak to more than $10 \mathrm{~ns}$ after peak temperature. Figure $6 \mathrm{c}$ shows the primary wall re-emission spectra for three different times in the temperature history. Two non-Planckian features are observed. Arrows note the positions of $\mathrm{x}$-ray absorption edges at $300 \mathrm{eV}$ and $500 \mathrm{eV}$ in $\mathrm{C}$ and $\mathrm{O}$ layers, probably from aperture blowoff during the pinch run-in phase. Excluding the data points at 300 and $500 \mathrm{eV}$ in the Planckian fit would raise the temperature by only $2.4 \%$ to $117.5 \mathrm{eV}$ (light solid line). Later in the pulse $\left(\mathrm{T}_{\mathrm{pk}}+10 \mathrm{~ns}\right)$ we also observe a general absorption below the Planckian fit between about 350 and $600 \mathrm{eV}$. This could be absorption in aperture plasma or absorption in $\mathrm{W}$ in the primary hohlraum. Secondary wall spectra are more Planckian, for up to $20 \mathrm{~ns}$ after peak. Z-pinch driven hohlraums are wellbehaved and compatible with the long drive times required for large capsules.

Figure 7 is a filtered $x$-ray framing camera picture $(>800 \mathrm{eV})$ of the Be spokes self-backlit by the primary hohlraum radiation field at peak axially-directed power. A secondary was not used in this experiment. The spokes are observed to maintain their spoke-like character through and after peak power, thus indicating the potential for high average transparency to $\mathrm{x}$-rays in between the spoke material as we have observed. A direct measurement of axial power in this configuration yielded $28 \pm 6 \mathrm{TW}$. A hohlraum reduces the net radiation transport $\left(\mathrm{T}_{\mathrm{p}}{ }^{4}-\mathrm{T}_{\mathrm{s}}{ }^{4}\right)$, because of a hotter secondary, however a hohlraum would also integrate over pinch direct shine, which axially-directed diagnostics do not observe. The 0-D energetics and 2-D VF models predict of order 9 TW direct shine, and 17 TW from radiative coupling, for $26 \pm 5$ TW total. We 
observe an additional low-temperature stagnation feature in between each spoke. This feature is discussed below.

Figure 8 gives the results of a 2-D RMHD simulation of the Be spoke transparency including the effects of ablation of spoke material by radiation and current drive. A high transparency of $75 \%$ is predicted for low- $Z$ Be spokes in agreement with the Be spoke transparency measured above of $71 \pm 7 \%$ (0-d model, when corrected for Au wall motion). As noted, we also observe a stagnation feature in between each spoke. Simulations with high $Z$ spoke material ${ }^{5}$ or with high $\mathrm{Z}$ contaminants in the bulk of the spoke show this inter-spoke stagnation feature at the midpoint between spokes. Experimentally this feature does not appear to significantly reduce the average spoke transparency and could be due to carbon, oxygen or other contaminants. This feature requires further study.

\section{Radiation symmetry capability: single-sided power feed, double-pinch x-ray source}

Figure 9 shows results from the double-pinch geometry depicted in Fig. 2C. A $15 \mathrm{~mm}$ long secondary is positioned within the middle of a $35 \mathrm{~mm}$ long wire array, creating two separate arrays above and below the seconday. The upper pinch is driven by a transmission line feed extended along the outside of the secondary. Current does not flow on the inside of the secondary because the Be spokes act as Faraday shields. The wires are removed from the secondary diagnostic apertures. We measured the upper and lower pinch powers and upper and lower primary temperatures in this experiment. These temperatures and powers are identical within the experimental errors of about \pm 20 to $\pm 27 \%$ in x-ray flux. The total power (energy) decreased from of order $115 \mathrm{TW}(1000 \mathrm{~kJ})$ for a single pinch, to of order $85 \mathrm{TW}(850 \mathrm{~kJ})$ on double pinch shots because the larger load inductance results in about 1-2 MA of increased 
electron loss in the transmission line feed. The primary temperatures are decreased to about 90 $\mathrm{eV}$ from the $120 \mathrm{eV}$ observed in single-sided case, because the effective hohlraum wall area has been increased by about $50 \%$ for each primary, the pinch power per primary is down by a factor of 2 , and because the total pinch power has been reduced. We understand the energetics and secondary coupling of the system to the $\pm 20 \%$ level in flux as in the single-sided case ${ }^{10}$. The upper primary has less radiation loss than the lower primary because it has only a single AK gap feed rather than two. A 0-D energetics model shows that the upper pinch power must be about $20 \%$ lower than the lower power to obtain identical primary temperatures ${ }^{10}$. However, Fig. 10 indicates that the upper temperature may be lower than the lower primary temperature for approximately equal powers. A possible explanation (other than measurement uncertainty) is that the upper pinch FWHM (6.8 ns) is smaller than the lower ( $8.2 \mathrm{~ns})$. This would result in lower albedo and lower temperature for the upper primary. The difference in FWHM results because the apertures in the lower primary were located more asymmetrically than in the upper primary (see Fig. 2C, compare aperture layout for $\mathrm{P}_{\mathrm{up}}$ and $\mathrm{P}_{\text {low }}$ ). This hypothesis was verified by switching the diagnostic locations between the top and bottom primaries (among other tests), which broadened the upper and narrowed the lower pulse.

The $\mathrm{P} 1$ radiation asymmetry that a capsule will see depends on the pinch power and temperature balance top-to-bottom as well as the relative timing of the peak pinch power. Capsule sensitivity studies are underway to place limits on tolerable capsule P1 drive. 2-D timedependent viewfactor simulations assuming the same limit for P1 as for P2 for the NIF capsule require that the pinch power balance be within $7 \%$ for identically shaped pulses, and the peak of such pulses be within $\pm 1 \mathrm{~ns}^{5.11}$. The peak of the upper pinch power was $0.22 \pm 0.20 \mathrm{~ns}$ prior to the peak of the lower power for the above shot. More than $2 / 3$ of recent shots show a timing 
difference between the peak of top and bottom powers within the \pm 1 ns window. The timing performance continues to improve as we optimize and match the top and bottom pulseshape and power balance by controlling aperture perturbations, wire array concentricity, double pinch fabrication and wire positioning and tensioning technique. The assessment of power balance topto-bottom for this shot is at about the limit of what is possible, given the uncertainties of local temperature and pinch measurements as discussed throughout the paper. Determining acceptable pinch balance for this configuration at the $5-10 \%$ percent level must be diagnosed with laserbacklit capsule implosions.

Since the mass of the upper and lower arrays are identical, implosion times within $\pm 1 \%$ as observed imply that there is very little current loss $(<250 \mathrm{kA}$ from $0-\mathrm{D}$ circuit simulations $)$ in the connecting transmission line, at least in the $100 \mathrm{~ns}$ up through peak power. After peak power however, the upper pinch power decreases faster than the lower pinch power. We also observe less radiated energy from the upper pinch compared to the lower pinch by about $20 \pm 10 \%$ where the two pulses have similar shapes and FWHM. The late time pulseshape difference and upper energy deficit are observed on all double pinch shots, independent of diagnostics used, aperture location, and AK gap. This suggests AK gap closure is affecting power output from the upper pinch and will cause a P1 swing later in time, e.g. Fig. 9 shows that the upper primary temperature is decreasing faster than the lower primary. Symmetry and implosion experiments with drive times longer than $15 \mathrm{~ns}$ after peak temperature will need to compensate for this $\mathrm{P} 1$ swing. This Pl swing and AK closure would not be an issue for the proposed high-yield geometry of Fig. 1, with a transmission line power feed for each pinch.

\section{Summary}


The experiments described in this paper characterized the pinch performance, hohlraum temperatures, energetics balance and secondary coupling for the ZPDH and compared the measurements with a variety of 2-D models. These experiments used all three configurations shown in Fig. 2. Pinch power was characterized in several different current return electrode geometries. Sensitivity of power and energy to aperture location, and wire positioning and tensioning technique was noted at the $15-25 \%$ level (1-2 ns). These effects are under investigation. Be spokes do not perturb the pinch pulseshape or implosion time. AK gap loss was indirectly inferred at the base of the pinch. 2-D E-RMHD codes do a good job of predicting the radiated energies (of order $0.9 \mathrm{MJ}$ during the main pulse for these pinches) and are able to match the $\mathrm{x}$-ray power and pulseshape under many circumstances by varying a random seed to represent effective initial condition for instability growth. Pinch measurements such as energy spectrum and compression ratio are poorly modeled with existing 2-D codes. In addition a number of second order 3-D pinch effects ( $r-\theta$ plane physics) on FWHM have been observed depending on aperture location and size and wire positioning and tensioning which are being characterized and optimized empirically. 3-D models of pinch performance and implosion physics should be developed.

The balance between the measured pinch powers and hohlraum temperatures is well understood from 0-D semi-analytic, 2-D viewfactor, and 2-D RMHD simulations to within the $\pm 20 \%$ uncertainty in measured $x$-ray flux. The modeling uncertainty in pinch and hohlraum energetics from several factors such as non-Lambertian. and non-uniform pinch emission, the contribution to pinch diagnostics from hohlraum wall re-emission and reflection off of the pinch, AK gap closure, and hohlraum temperature history during the pinch run-in phase are all bounded by the flux uncertainty. We show high Be-spoke transparency $(>71 \pm 7 \%)$ in good agreement with 
2-D RMHD simulations (75\%). This is a level sufficient for high-yield 5 . These same simulations show that $75 \%$ transparency can be achieved for high yield conditions ${ }^{5}$. Scaling to high-yield temperatures will be possible if the pinch power and AK gap performance can be scaled to of order 1000-1200 TW, depending on the amount of AK gap closure. We have also developed a single-sided power feed, double-pinch capability required for polar radiation symmetry and capsule implosion on $\mathrm{Z}$. This source has demonstrated the required pinch simultaneity for control of capsule $\mathrm{Pl}$ and progress has been made towards the required pinch balance and radiation symmetry. This source has the potential to be the first pulsed power device capable of demonstrating ICF relevant radiation symmetry. We noted in the introduction that one of the potential benefits of the ZPDH approach is that the 3-D Z-pinch source region physics was decoupled from the capsule physics, promising an approach that is more readily modeled with available 2-D codes, and therefore has improved predictability to scale the system. The excellent agreement between the various 2-D energetics and transparency models and data have fullfilled this promise.

Future experiments with concentrate on backlit implosions to directly measure radiation symmetry at a capsule in the period before the AK gap loss produces a P1 swing. This capability will permit us to infer the statistical probability of achieving pinch power balance within $7 \%$ (meeting high yield requirements) and also allow study of geometric symmetry control techniques ${ }^{11}$. Demonstration of an effective top-to-bottom temperature balance within $2 \%$ will meet pinch power balance requirements to minimize P1. If the late-time P1 swing can be compensated for or controlled, demonstration of a capsule convergence ratio of 7 at double-pinch secondary temperatures on $\mathrm{Z}(75 \pm 5 \mathrm{eV})$ would demonstrate a time-integrated RMS capsule radiation symmetry of about $4 \%$, which would scale to the requisite $1 \%$ at high yield temperatues 
$(210 \mathrm{eV})$. Experiments addressing AK gap closure and its affect on radiation trapping, and pinch power scaling with current are planned this year. We have begun developing capsule preheat diagnostic techniques. Pulseshaping must also be addressed in a systematic way in the future.

\section{Acknowledgements}

We gratefully acknowledge the technical efforts of the $\mathrm{Z}$ operations teams led by J. Seamen, including machine operations, data acquisition (J. Mills), the wire array lab (K. Tapia, D. Graham), target development lab (D. Hebron, S. Dropinski), and materials processing and coating lab (J. McKenney). We also thank P. Primm and T. Wagoner for technical assistance. We acknowledge helpful discussions with R. Chrien, C. Deeney, N. Landen, T. Nash, and A. Toor. We thank M. K. Matzen and J. P. Quintenz for programmatic support. Sandia is a multiprogram laboratory operated by Sandia Corporation, a Lockheed Martin Company, for the U. S. Department of Energy under Contract No. DE-AC04-94AL85000.

\section{References}

1. T. W. L. Sanford, G. O. Allshouse, B. M. Marder, et al., Phys. Rev. Lett., 77, 5063 (1996).

2. C. Deeney, T. J. Nash, R. B. Spielman, et al., Phys. Rev. E, 56, 5945 (1997).

3. R. B. Spielman, C. Deeney, G. A. Chandler, et al., Phys. Plasmas, 5, 2105 (1998).

4. J. D. Lindl, Phys. Plasmas, 2, 3933, (1995).

5. J. H. Hammer, M. Tabak, S. C. Wilks, et al., Phys. Plasmas, 6, 2129(1999).

6. J. S. Lash, G. Chandler, G. Cooper, et al., Inertial Fusion Science and Applications 99, Eds. C. Labaune, W. J. Hogan, K. A. Tanaka, (Elsevier, Paris, 2000), p. 583.

7. T. W. L. Sanford, R. E. Olson, R. L. Bowers, et al., Phys. Rev. Lett., 83, 551 (1999).

S. M. Rosen, Phys. Plasmas, 3, 1803 (1996).

9. J. L. Porter, Jr., Bull. Amer. Phys. Soc., 42, 1948 (1997). 
10. M. E. Cuneo, R. A. Vesey, J. H. Hammer, J. L. Porter, Jr., L. E. Ruggles, W. W. Simpson, "0-d energetics models for Z-pinch driven hohlraums," submitted to Lasers and Particle Beams.

11. R. A. Vesey, M. E. Cuneo, D. L. Hanson, et al., Bull. Amer. Phys. Soc., 44, 227(1999); Bull. Amer. Phys. Soc., this issue.

12. R. E. Chrien, W. Matuska, Jr., F. J. Swenson, et al., Rev. Sci. Inst., 70, 557(1999).

13.L. E. Ruggles, M. E. Cuneo, J. L. Porter, et al., "Measurement of the efficiency of gold transmission gratings in the 100 to $5000 \mathrm{eV}$ photon energy range", submitted to Rev. Sci. Inst.

14. G. A. Chandler, C. Deeney, M. Cuneo, et al., Rev. Sci. Inst., 70, 561(1999).

15. G. C. Idzorek, R. J. Bartlett, in Proceedings of the $11^{\text {th }}$ Inter. Pulsed Power Conf., Baltimore, MD, 1997, G. Cooperstein and I. Vitkovitsky, Eds., Vol. 2, p. 1274.

16. R. B. Spielman, C. Deeney, D. L. Fehl, et al., Rev. Sci. Inst., 70, 651(1999).

17. W. A. Stygar, R. B. Spielman, H. C. Ives, et al., in Proceedings of the $11^{\text {th }}$ Inter. Pulsed Power Conf., Baltimore, MD, 1997, G. Cooperstein and I. Vitkovitsky, Eds., Vol. 2, p. 1258.

18. J. H. Hammer, D. D. Ryutov, Phys. Plasmas, 6, 3302(1999).

19. D. L. Peterson, R. L. Bowers, K. D. McLenithan, et al., Phys. Plasmas, 5, 3302(1998).

20. D. L. Peterson, R. L. Bowers, W. Matuska, et al., Phys. Plasmas, 6, 2178(1999).

21. G. B. Zimmerman and W. L. Kruer, Comments Plasma Phys. Control. Fusion, 2, 51 (1975). 


\section{Tables}

Table I. Pinch energetics characterization'

\begin{tabular}{|c|c|c|c|c|}
\hline $\begin{array}{c}\text { Can Configuration } \\
\text { Shots }\end{array}$ & $\mathrm{EBOL}(\mathrm{kJ})$ & $\begin{array}{c}\text { PXRDshape/BOL (TW) } \\
\text { FWHM (ns) }\end{array}$ & $\begin{array}{l}\text { PXRD (TW) } \\
\operatorname{EXRD~(kJ)~} \\
\text { FWHM (ns) }\end{array}$ & $\begin{array}{l}\text { PTGS (TW) } \\
\text { ETGS (kJ) } \\
\text { FWHM (ns) }\end{array}$ \\
\hline $\begin{array}{l}\text { Slotted/no spokes } \\
566\end{array}$ & $1146 \pm 172$ & $\begin{array}{c}117 \pm 29 \\
6.3\end{array}$ & $\begin{array}{c}127 \pm 19 \\
1140 \\
5.9\end{array}$ & $\begin{array}{c}105 \pm 26 \\
1330 \pm 333 \\
7.7\end{array}$ \\
\hline $\begin{array}{c}\text { Slotted/spokes } \\
567\end{array}$ & $1035 \pm 155$ & $\begin{array}{c}104 \pm 26 \\
6.7\end{array}$ & $\begin{array}{l}97 \pm 15 \\
907 \\
6.4\end{array}$ & $\begin{array}{c}105 \pm 26 \\
1160 \pm 290 \\
7.0\end{array}$ \\
\hline $\begin{array}{l}\text { Primary/no spokes }{ }^{2} \\
\text { aperture setup } A^{3} \\
272,273,274,275.319\end{array}$ & $\begin{array}{c}1163 \\
( \pm 27)( \pm 209)\end{array}$ & $\begin{array}{c}131( \pm 7)( \pm 37) \\
6.4 \pm 0.5\end{array}$ & not available & not available \\
\hline $\begin{array}{l}\text { Primary/Secondary/spokes }{ }^{2} \\
\text { aperture setup } \mathrm{Cl}^{4} / \text { spreader }^{2} \\
413,415,500\end{array}$ & $\begin{array}{c}1043 \\
( \pm 18)( \pm 188)\end{array}$ & $\begin{array}{c}99( \pm 7)( \pm 27) \\
7.7 \pm 0.3\end{array}$ & not available & not available \\
\hline
\end{tabular}

(1) first error in parenthesis is shot-to-shot, second is absolute

(2) hohlraum numbers have been aperture closure corrected at peak 


\section{Figure Captions}

Figure 1. High yield ICF Z-pinch driven hohlraum configuration showing two $24 \times 10 \mathrm{~mm}^{2}$ primaries or x-ray source hohlraums each driven by $300,11.5 \mu \mathrm{m}$ W wire arrays, coupled to a $20 \times 15 \mathrm{~mm}^{2}$ secondary with a $5 \mathrm{~mm}$ diam. high yield capsule.

Figure 2. Experimental and diagnostic line-of-sight (LOS) layout showing: A) single-sided secondary with either a slotted or solid return current electrode (high yield configuration in dotted lines), B) primary only with either a slotted or solid return current electrode and C) singlesided power feed, double-sided pinch configuration showing: (a) transmission line feed, (b) anode-cathode (AK) gap at the base of the pinch, (c) W wire array at initial position (dotted line), (d) x-ray picture of stagnated pinch with 2-3 mm FWHM, (e) shine shield for capsule symmetry, (f) Be spokes, (g) LOS with XRD/BOL/FSDA, (h) LOS with TGS/FC, (i) LOS with TGS/FC, (j) LOS with XRD/BOL/TGS, (k) LOS with FC, (1) LOS with ISDA. Note the various primary (P, Pslots, Pup, Plow) and secondary (S) views.

Figure 3. Comparison of pinch power pulse shape for five different experimental geometries using a $20 \mathrm{~mm}$ wire array with $300,11.5 \mu \mathrm{m}$ wires, comparing the effects of aperture geometry, spokes, and wire positioning. $\mathrm{P}$ indicates primary as in Fig. 2B), P/S primary/secondary as in Fig. 2A. Primary aperture setups are as follows: A $\left(4 \mathrm{~mm} \times 4 \mathrm{~mm}\right.$ at $50^{\circ}$, a pair of $2.4 \mathrm{~mm} \phi$ at $110^{\circ}, 3$ $\mathrm{mm} \phi$ covered by $10 \mu \mathrm{m} \mathrm{CH}$ at $\left.350^{\circ}\right)$, B: $\left(4 \mathrm{~mm} \mathrm{x} 4 \mathrm{~mm}\right.$ at $50^{\circ}, 3 \mathrm{~mm} \phi$ at $\left.195^{\circ}\right)$. $\mathrm{Cl}(4 \mathrm{~mm} \mathrm{x} 4$ $\mathrm{mm}$ at $50^{\circ}, 4 \mathrm{~mm} \phi$ at $\left.145^{\circ}\right), \mathrm{C} 2\left(6 \mathrm{~mm} \times 4 \mathrm{~mm}\right.$ at $50^{\circ}, 4 \mathrm{~mm} \phi$ at $\left.145^{\circ}\right) . \mathrm{P} / \mathrm{S}$ shots use wire spreader hardware to move wires out of the way of secondary apertures. Shot numbers are given in parenthesis. 
Figure 4. Comparison of current and power measurements for shot 566 with the results of 2-D Eulerian radiation-MHD simulations, with and without AK gap power loss showing the measured total $\left(I_{\text {total }}\right)$ and post-convolute currents $\left(I_{\text {conv }}\right)$, the 2-D convolute and load currents with AK loss $\left(I_{\text {comn2D }}, I_{\text {load2D }}\right)$, the 0-D load current with loss $\left(I_{\text {loadoD }}\right)$, the 2-D load current without loss $\left(I_{\text {loadnoloss }}\right)$, the measured pinch power $\left(P_{\text {XRD566 }}\right)$, the 2-D pinch power with loss $\left(P_{2-D}\right)$ and the 2-D pinch power without loss $\left(\mathrm{P}_{\text {noloss }}\right)$. The peak pinch power is at $225 \mathrm{~ns}$.

Figure 5. Pinch spectral power distribution from transmission grating spectrometer unfolds (black circles with error bars and solid line Plankian fit) for shot 567, compared with predictions from a 2-D E-RMHD pinch simulation. The 2-D RMHD simulation results are post-processed to produce a synthetic TGS spectrum which is well represented by the sum of two Planckian distributions, each Planckian with its own temperature and area.

Figure 6. Comparison of measurements from a single-sided secondary shot 413 with 2-D radiation-MHD and 2-D viewfactor models showing a) measured primary temperature ( $\left.\mathrm{T}_{\mathrm{pTGS}}\right)$ and pinch power $\left(\mathrm{P}_{\mathrm{p} X R D / B O L}\right)$ compared with a viewfactor model $\left(\mathrm{T}_{\mathrm{pVF}}\right.$ using $95 \mathrm{TW}$ and $65 \%$ transparency) and two 2-D RMHD models ( $\mathrm{T}_{\mathrm{pRMHD}}$ with $95 \mathrm{TW} / 72 \%$ and $\left.106 \mathrm{TW} / 65 \%\right)$. The 2D models use either the measured power pulse shape (106 TW) or a scaled version ( $\mathrm{P}_{\mathrm{p} 2 \mathrm{D}}$ at $\left.95 \mathrm{TW}\right)$, b) measured secondary temperatures with three diagnostics $\left(T_{\mathrm{sTGS}}, T_{\mathrm{sFSDA}}, \mathrm{T}_{\mathrm{sPIN} / \mathrm{BOL}}\right)$ compared with the same three models, c) wall re-emission spectra and Planckian best-fits for primary hohlraum for three times ( $10 \mathrm{~ns}$ prior to peak temperature, at peak and 10 ns after peak). Arrows labeled $\mathrm{C}$ and $\mathrm{O}$ show the location of $\mathrm{x}$-ray absorption edges of aperture plasma. The light solid line is a Planckian fit without the Si PIN detectors at the $\mathrm{C}$ and $\mathrm{O}$ edges. 
Figure 7. X-ray framing camera picture $(>800 \mathrm{eV})$ of a Be spoke array backlit by the primary hohlraum radiation field, at peak axially-directed power on shot 319 .

Figure 8. 2-D RMHD simulation of the transparency of a Be spoke array with 18 spokes including the effects of current and radiation-driven ablation. This simulation is orientated looking radially down a single spoke towards the pinch axis, with mirror boundary conditions at either edge. The Be transparency is predicted to be $75 \%$ at $\mathrm{Z}$ conditions.

Figure 9. Upper and lower primary wall temperatures and pinch powers for double pinch shot 609. 
Figures 


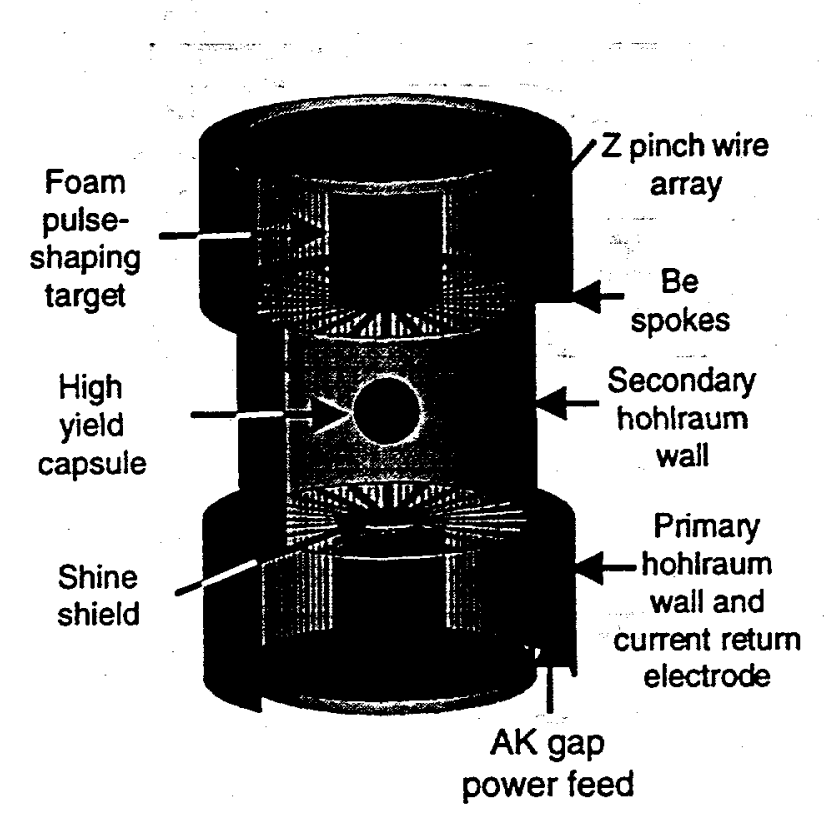

Figure 1. Cuneo et al., Phys. Plasmas 


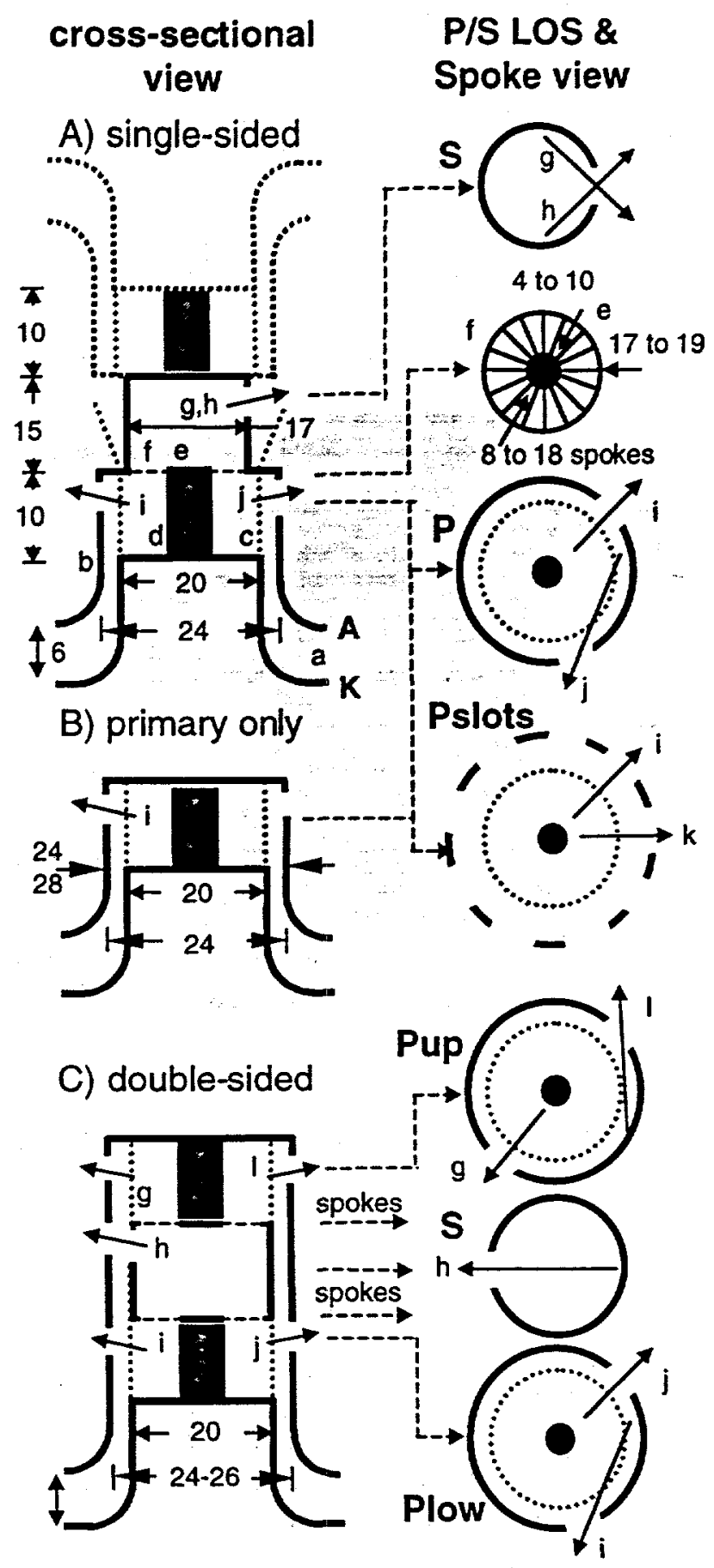

(all dimensions in $\mathrm{mm}$ )

Figure 2. Cuneo et al., Phys. Plasmas 


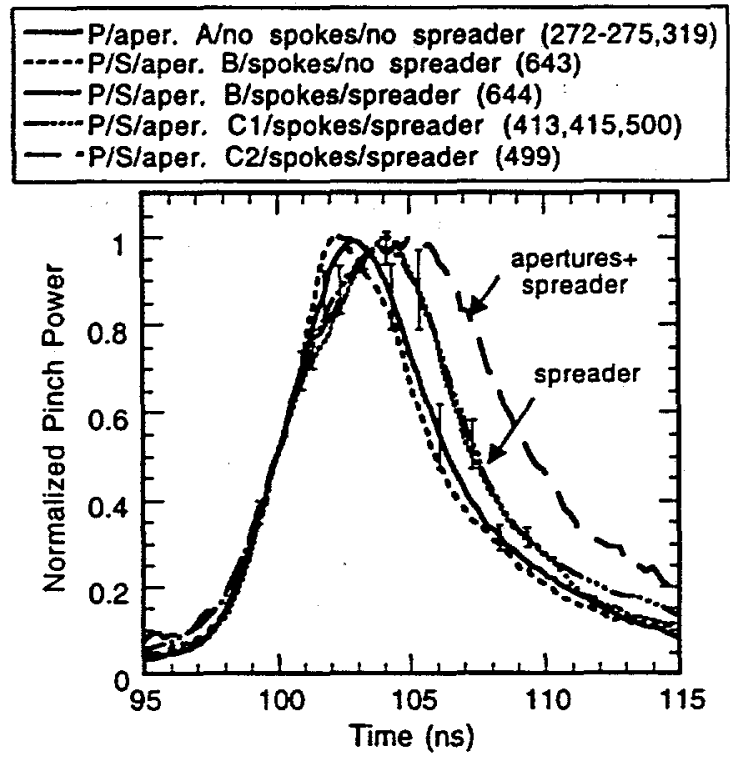

Figure 3. Cuneo et al., Phys. Plasmas 


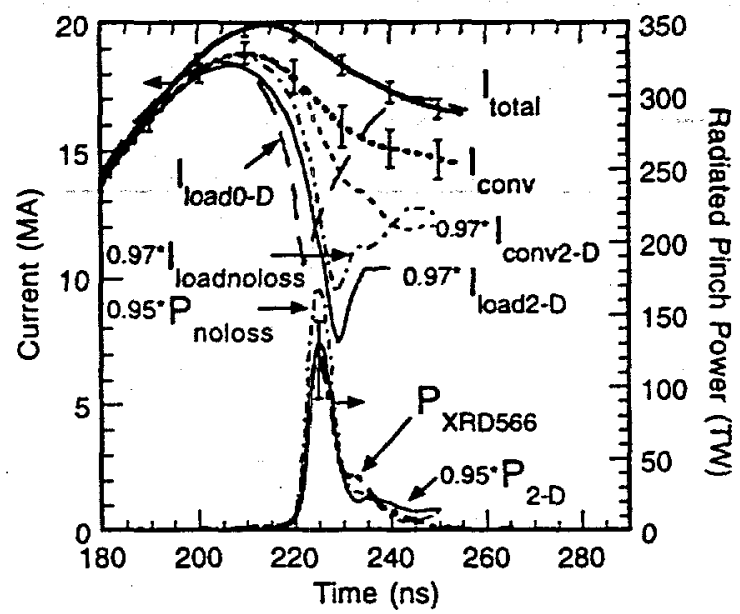

Figure 4. Cuneo et al., Phys. Plasmas 


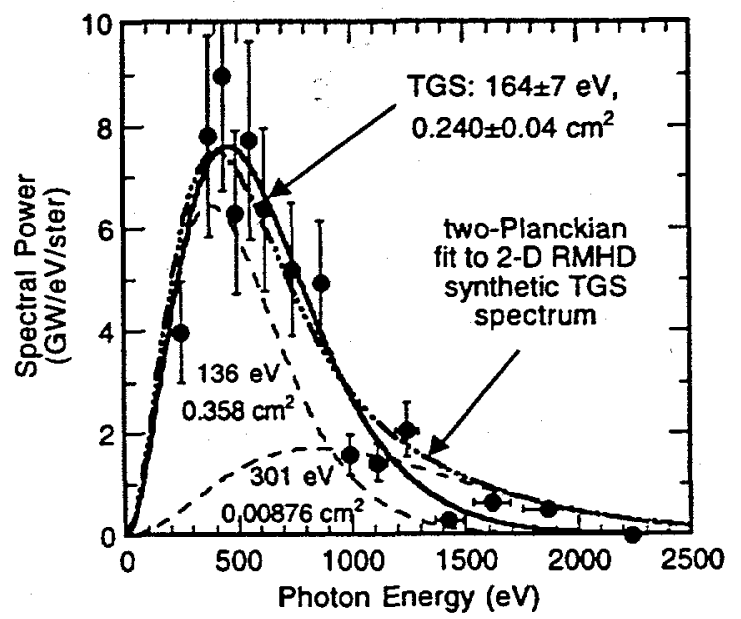

Figure 5. Cuneo et al., Phys. Plasmas 


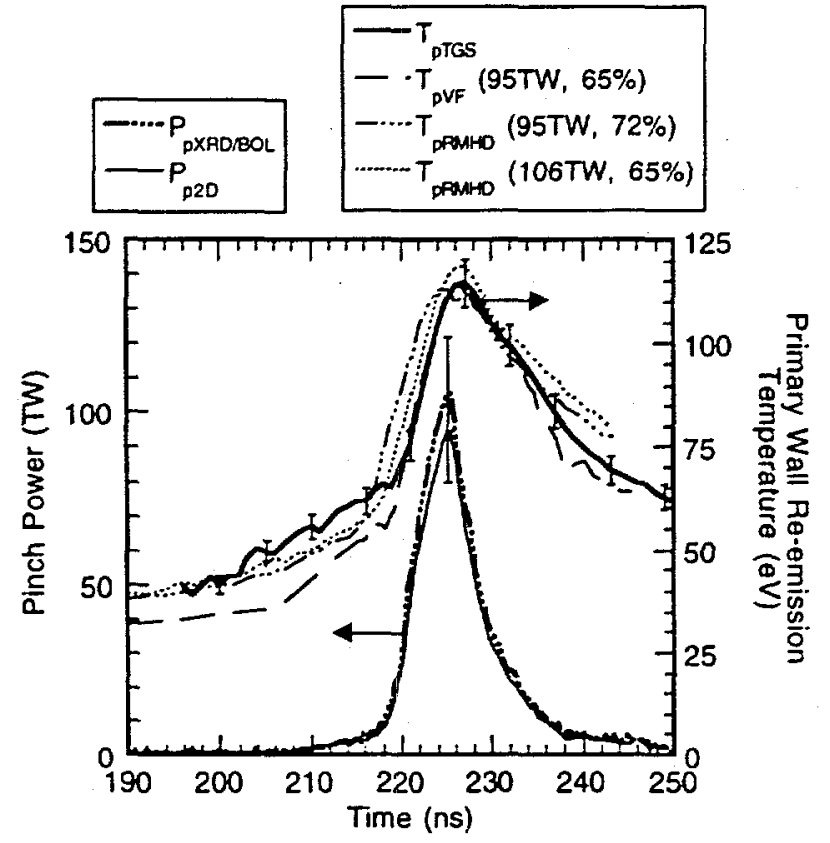

Figure 6a. Cuneo et al., Phys. Plasmas 


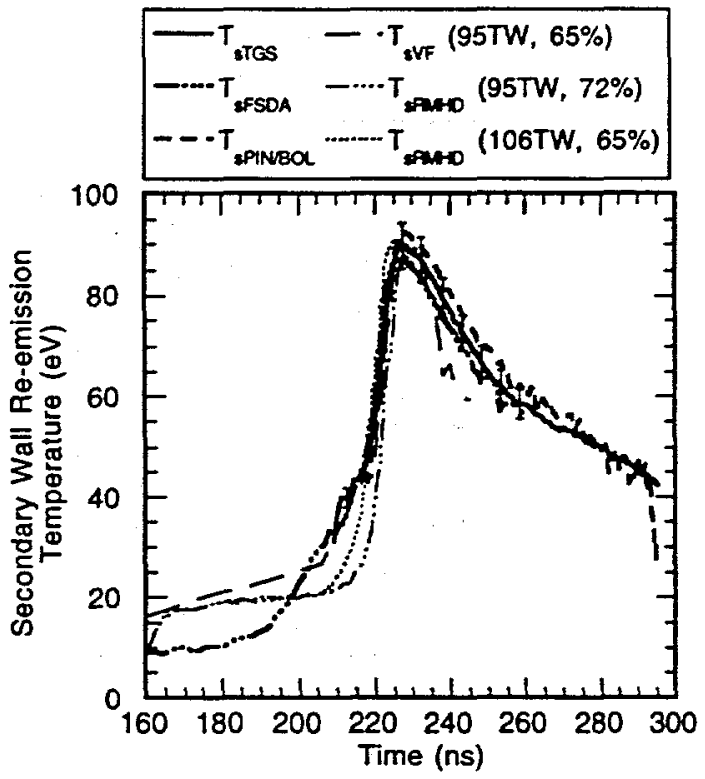

Figure 6b. Cuneo et al., Phys. Plasmas 


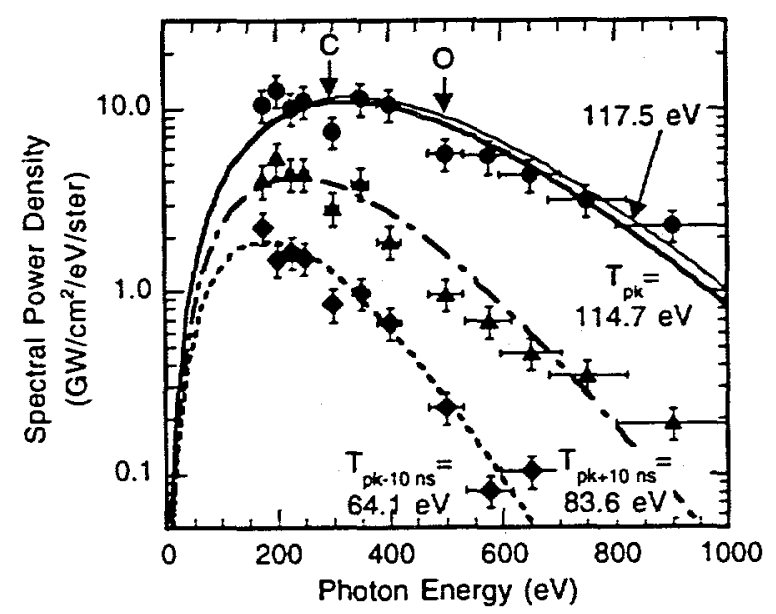

Figure 6c. Cuneo et al., Phys. Plasmas 


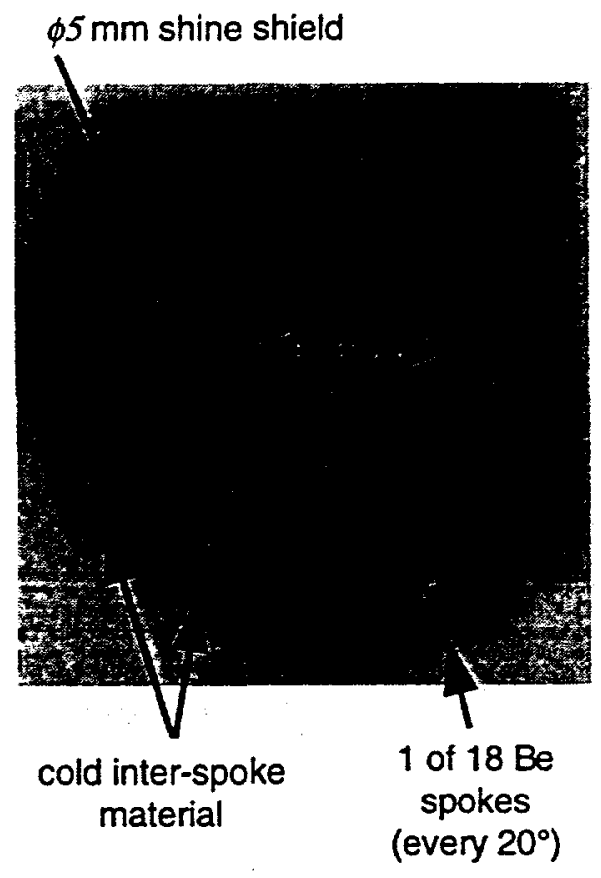

Figure 7. Cuneo et al., Phys. Plasmas 



Rosseland

mean free path

contours

Figure 8. Cuneo et al., Phys. Plasmas 


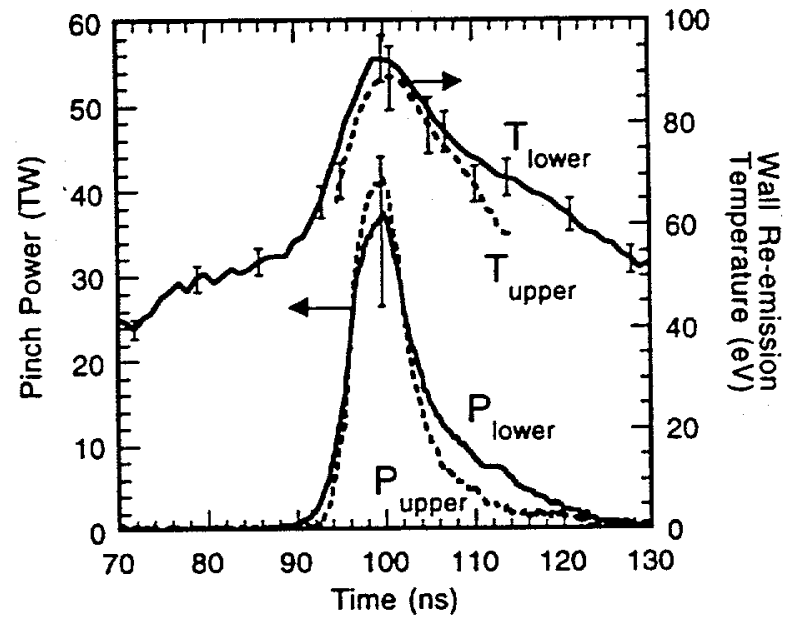

Figure 9. Cuneo et al., Phys. Plasmas 